\title{
Jet quenching in a strongly interacting plasma - A lattice approach
}

\author{
Marco Panero* \\ Department of Physics and Helsinki Institute of Physics, University of Helsinki, \\ P.O. Box 64, FI-00014 Helsinki, Finland \\ E-mail: marco.panero@helsinki.fi

\section{Kari Rummukainen} \\ Department of Physics and Helsinki Institute of Physics, University of Helsinki, \\ P.O. Box 64, FI-00014 Helsinki, Finland \\ E-mail: kari.rummukainen@helsinki.fi
}

\section{Andreas Schäfer}

Institute for Theoretical Physics, University of Regensburg, D-93040 Regensburg, Germany

E-mail: andreas.schaefer@physik.uni-regensburg.de

The phenomenon of jet quenching, related to the momentum broadening of a high-energy parton, provides important experimental evidence for the production of a strongly coupled, deconfined medium in heavy-ion collisions. Its theoretical description has been addressed in a number of works, both perturbatively and non-perturbatively (using the gauge-gravity duality). In this contribution, following a proposal by Caron-Huot, we discuss a novel approach to this problem, enabling one to extract non-perturbative information on this real-time phenomenon from simulations on a Euclidean lattice.

The European Physical Society Conference on High Energy Physics

18-24 July, 2013

Stockholm, Sweden

\footnotetext{
* Speaker.
} 


\section{Introduction}

More than thirty years ago, Bjorken suggested a possible way to detect the creation of deconfined QCD matter in collisions of ultrarelativistic nuclei: due to interactions with the medium constituents, a hard parton propagating through the quark-gluon plasma (QGP) at a given temperature $T$ would experience energy loss and momentum broadening, and this would result in the suppression of final-state hadrons with large transverse momentum and of back-to-back correlations [1]. This prediction was eventually confirmed by experiments [2].

Providing a firm theoretical description for this beautiful physical idea is, however, challenging, as it involves an interplay of both perturbative and non-perturbative physics effects [3]. Even if one focuses only on the short-distance interactions between the hard parton and QGP constituents [4], the problem is still complicated by the fact that, for temperatures within the reach of present experiments, the QCD coupling $g$ is not very small, so perturbative computations may not be reliable. On the other hand, strong-coupling approaches based on the gauge/string duality, like the ones carried out for a massless hard parton [5] or for the drag force experienced by a heavy quark [6], are not based on the QCD Lagrangian. Finally, non-perturbative lattice QCD computations are not straightforward for this real-time problem.

In this contribution, however, we would like to discuss some recent progress in the latter direction [7], based on an idea proposed in ref. [8]. Related studies include refs. [9-11], whereas a different way to study the jet quenching phenomenon on the lattice was proposed in ref. [12].

\section{Soft contribution to jet quenching from a Euclidean lattice}

Jet quenching can be described in terms of a phenomenological parameter $\hat{q}$, defined as the average increase in the squared transverse momentum component $p_{\perp}$ of the hard parton per unit length. This quantity can be expressed in terms of a differential collisional rate between the parton and plasma constituents $C\left(p_{\perp}\right)$ :

$$
\hat{q}=\frac{\left\langle p_{\perp}^{2}\right\rangle}{L}=\int \frac{\mathrm{d}^{2} p_{\perp}}{(2 \pi)^{2}} p_{\perp}^{2} C\left(p_{\perp}\right) .
$$

In turn, $C\left(p_{\perp}\right)$ is related to the two-point correlation function of light-cone Wilson lines. Although the full computation of this correlator cannot be carried out on a Euclidean lattice, it is possible to extract the non-perturbative contributions to it from the soft sector, i.e. from physics at momentum scales up to $g T$, which can be proven to be time-independent $[8,9]$. Evaluating the non-perturbative contribution from soft (and ultrasoft, of order $g^{2} T / \pi$ ) modes is important, since they are responsible for the peculiar analytical structure of weak-coupling computations in thermal QCD and for the large corrections affecting the corresponding perturbative series. A proper systematic framework to deal with these problems can be formulated in terms of dimensionally reduced effective theories [13]. In particular, the soft-scale dynamics can be described by electrostatic QCD (EQCD): an effective theory for the static QGP modes, given by three-dimensional Yang-Mills theory coupled to an adjoint scalar field,

$$
\mathscr{L}=\frac{1}{4} F_{i j}^{a} F_{i j}^{a}+\operatorname{Tr}\left(\left(D_{i} A_{0}\right)^{2}\right)+m_{\mathrm{E}}^{2} \operatorname{Tr}\left(A_{0}^{2}\right)+\lambda_{3}\left(\operatorname{Tr}\left(A_{0}^{2}\right)\right)^{2} .
$$




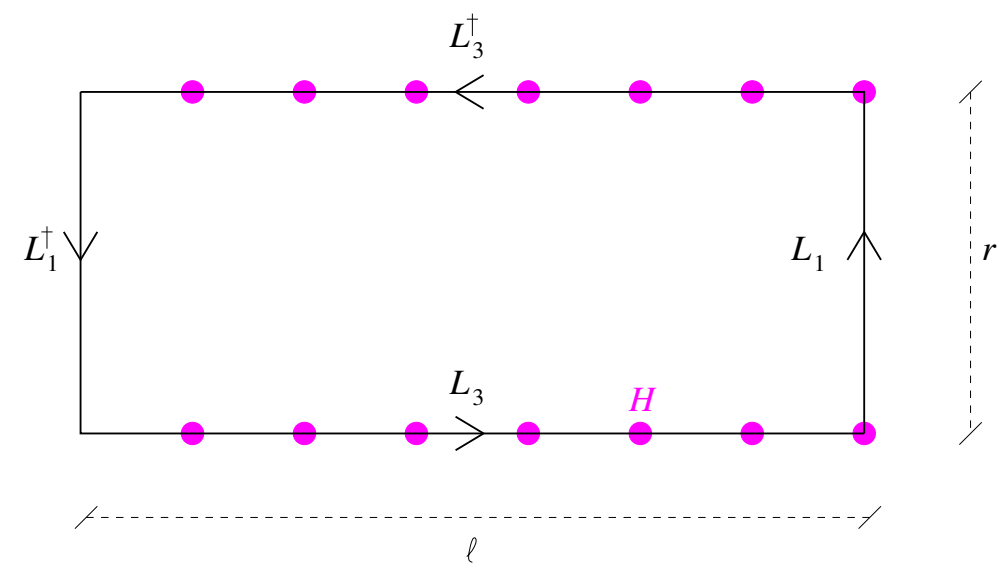

Figure 1: The "decorated" Wilson loop $W(\ell, r)$ describing a two-point correlation function of light-cone Wilson lines involves Hermitian parallel transporters $H(x)$ along the real-time direction.

Its parameters (the 3D gauge coupling $g_{\mathrm{E}}$ and the mass- and quartic-term coefficients) can be fixed by matching to the physics of high-temperature QCD, and the theory can be regularized on a lattice. We chose a setup corresponding to the dimensional reduction of QCD with $n_{f}=2$ light dynamical quark flavors, at two temperatures $(T \simeq 398 \mathrm{MeV}$ and $2 \mathrm{GeV})$ approximately twice and ten times larger than the deconfinement temperature [14].

We simulate this theory and study (a gauge-invariant generalization of) the two-point correlator of light-cone Wilson lines, defined in terms of a lattice operator which involves parallel transporters $H(x)=\exp \left[-a g_{\mathrm{E}}^{2} A_{0}(x)\right]$ along real time, which are Hermitian-rather than unitary-operators. This results in a "decorated" Wilson loop $W(\ell, r)$ (see fig. 1) with well-defined renormalization properties [15]. From its expectation values (computed with the multilevel algorithm [16]) we extract a "potential"

$$
V(r)=-\frac{1}{\ell} \ln \langle W(\ell, r)\rangle,
$$

which is equal to the transverse Fourier transform of $C\left(p_{\perp}\right)$.

At short distances our results for $V(r)$ (shown in fig. 2) are compatible with perturbative expectations, which involve, in particular, a delicate cancellation between gluon and scalar propagators $[8,9]$. The non-perturbative contributions to $V(r)$ can be related to $\hat{q}$ : the latter is given by the second moment of the distribution associated with $C\left(p_{\perp}\right)$, which corresponds to curvature terms in $V(r)$. Following an approach similar to ref. [10], we arrive at quite large values for the soft NLO contribution to the jet quenching parameter: $0.55(5) g_{\mathrm{E}}^{6}$ for $T \simeq 398 \mathrm{MeV}$, and $0.45(5) g_{\mathrm{E}}^{6}$ for $T \simeq 2 \mathrm{GeV}$. In turn, these numbers lead to a final estimate for $\hat{q}$ around $6 \mathrm{GeV}^{2} / \mathrm{fm}$ for RHIC temperatures, comparable with those from holographic estimates [5] and from computations with phenomenological input [17].

\section{Conclusions and outlook}

We have shown that, contrary to naïve intuition, the lattice study of certain real-time phenomena involving physics on the light cone is possible. Here we have discussed the phenomenon of jet 

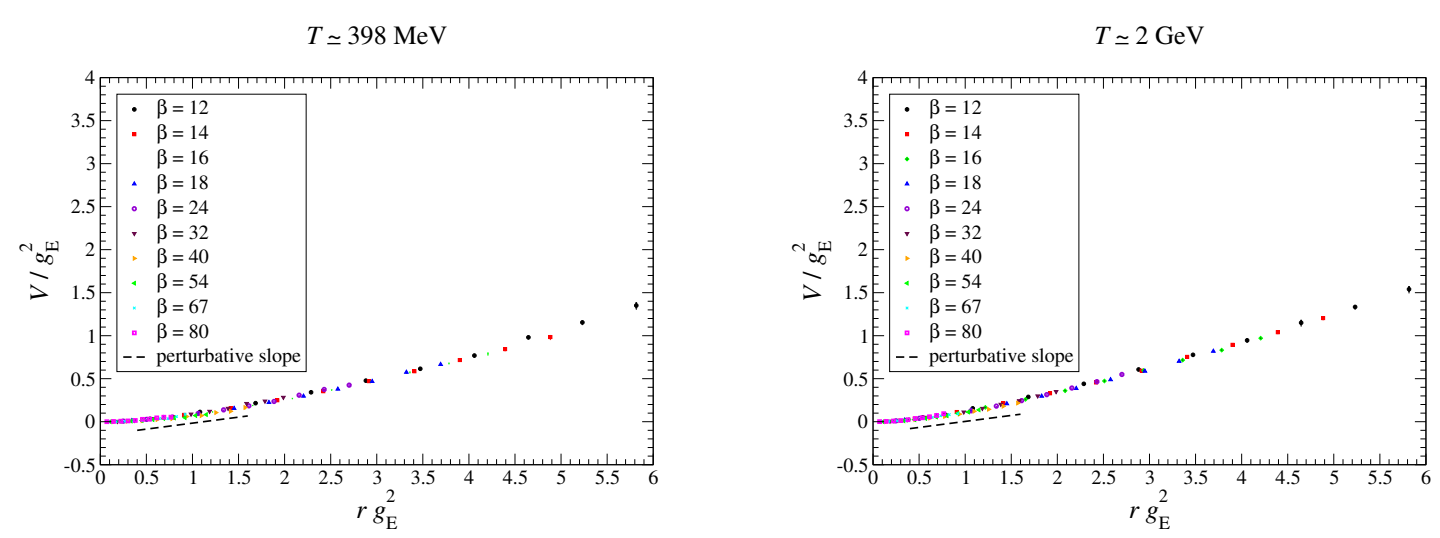

Figure 2: The "potential" $V(r)$ extracted from the expectation values of $W(\ell, r)$, at $T \simeq 398 \mathrm{MeV}$ (left-handside panel) and at $T \simeq 2 \mathrm{GeV}$ (right-hand-side panel). Both $V$ and $r$ are shown in the appropriate units of the dimensionful 3D gauge coupling $g_{\mathrm{E}}$. The slope predicted perturbatively for the potential at values $r g_{\mathrm{E}}^{2} \gtrsim 1$ is also displayed.

quenching in thermal QCD, but related ideas have also been proposed for QCD at zero temperature [18].

By construction, the bosonic effective theory that we simulated in our approach allows one to separate the soft contributions to $\hat{q}$ from those due to hard thermal modes, with momenta of order $\pi T$. It does so in a controlled, systematic way, consistent with the modern theoretical framework to study finite-temperature QCD [13, 19].

In the near future, we plan to improve our extrapolation of the potential $V(r)$ to the continuum limit at short $r$ by carrying out further simulations on finer lattices, and/or using improved actions [20]. It would also be interesting to study the dependence of $\hat{q}$ on $T$, and on the number of color charges $N$. As it is well-known, the large- $N$ limit is characterized by a rich and interesting phenomenology [21], and lattice studies have shown that the static equilibrium properties of the QGP have very little dependence on $N$, both in four [22] and in three [23] spacetime dimensions.

\section{Acknowledgements}

This work is supported by the Academy of Finland (project 1134018) and by the German DFG (SFB/TR 55). M.P. acknowledges financial support for travel from Università della Calabria (fund PAPA 340101 - Contributo Unical 2012 Progetto FIRB), Cosenza, Italy. Part of the numerical simulations was carried out at the Finnish IT Center for Science (CSC) in Espoo, Finland.

\section{References}

[1] J. D. Bjorken, Highly Relativistic Nucleus-Nucleus Collisions: The Central Rapidity Region, Phys. Rev. D 27 (1983) 140.

[2] K. Adcox et al. [PHENIX Collaboration], Suppression of hadrons with large transverse momentum in central Au+Au collisions at $\sqrt{s_{N N}}=130 \mathrm{GeV}$, Phys. Rev. Lett. 88 (2002) 022301

[nucl-ex/0109003]. I. Arsene et al. [BRAHMS Collaboration], Transverse momentum spectra 
in $A u+A u$ and $d+A u$ collisions at $\sqrt{s_{N N}}=200 \mathrm{GeV}$ and the pseudorapidity dependence of high $p(T)$ suppression, Phys. Rev. Lett. 91 (2003) 072305 [nucl-ex/0307003] . G. Aad et al. [ATLAS Collaboration], Observation of a Centrality-Dependent Dijet Asymmetry in Lead-Lead Collisions at $\sqrt{s_{N N}}=2.77 \mathrm{TeV}$ with the ATLAS Detector at the LHC, Phys. Rev. Lett. 105 (2010) 252303 [arXiv:1011.6182 [hep-ex] ]. K. Aamodt et al. [ALICE Collaboration], Suppression of Charged Particle Production at Large Transverse Momentum in Central $\mathrm{Pb}-\mathrm{Pb}$ Collisions at $\sqrt{s_{N N}}=2.76$ TeV, Phys. Lett. B 696 (2011) 30 [arXiv:1012.1004 [nucl-ex] ]. S. Chatrchyan et al. [CMS Collaboration], Observation and studies of jet quenching in $\mathrm{PbPb}$ collisions at nucleon-nucleon center-of-mass energy = 2.76 TeV, Phys. Rev. C 84 (2011) 024906 [arXiv:1102.1957 [nucl-ex]].

[3] J. Casalderrey-Solana and C. A. Salgado, Introductory lectures on jet quenching in heavy ion collisions, Acta Phys. Polon. B 38 (2007) 3731 [arXiv:0712.3443 [hep-ph] ] .

[4] R. Baier et al., Radiative energy loss of high-energy quarks and gluons in a finite volume quarkgluon plasma, Nucl. Phys. B 483 (1997) 291 [hep-ph/9607355].

[5] H. Liu, K. Rajagopal and U. A. Wiedemann, Calculating the jet quenching parameter from AdS/CFT, Phys. Rev. Lett. 97 (2006) 182301 [hep-ph/0605178].

[6] C. P. Herzog et al., Energy loss of a heavy quark moving through N=4 supersymmetric Yang-Mills plasma, JHEP 0607 (2006) 013 [ hep-th/ 0605158 ] . S. S. Gubser, Drag force in AdS/CFT, Phys. Rev. D 74 (2006) 126005 [hep-th/ 0605182 ] . J. Casalderrey-Solana and D. Teaney, Heavy quark diffusion in strongly coupled N=4 Yang-Mills, Phys. Rev. D 74 (2006) 085012 [ hep-ph/ 0605199 ] . U. Gürsoy et al., Thermal Transport and Drag Force in Improved Holographic QCD, JHEP 0912 (2009) 056 [arXiv:0906.1890 [hep-ph ] ] .

[7] M. Panero, K. Rummukainen and A. Schäfer, A lattice study of the jet quenching parameter, arXiv:1307.5850 [hep-ph]. See also M. Panero, K. Rummukainen and A. Schäfer, Momentum broadening of partons on the light cone from the lattice, POS (LATTICE 2013) 173 [arXiv:1309.3212 [hep-lat] ] for a concise summary.

[8] S. Caron-Huot, $O(g)$ plasma effects in jet quenching, Phys. Rev. D 79 (2009) 065039 [arXiv:0811.1603 [hep-ph] ].

[9] J. Ghiglieri et al., Next-to-leading order thermal photon production in a weakly coupled quark-gluon plasma, JHEP 1305 (2013) 010 [arXiv:1302.5970 [hep-ph] ] .

[10] M. Laine, A non-perturbative contribution to jet quenching, Eur. Phys. J. C 72 (2012) 2233 [arXiv:1208.5707 [hep-ph] ].

[11] M. Benzke et al., Gauge invariant definition of the jet quenching parameter, JHEP 1302 (2013) 129 [arXiv:1208.4253 [hep-ph] ]. M. Laine and A. Rothkopf, Light-cone Wilson loop in classical lattice gauge theory, JHEP 1307 (2013) 082 [ arXiv:1304.4443 [hep-ph] ]; Towards understanding thermal jet quenching via lattice simulations, POS (LATTICE 2013) 174. I. O. Cherednikov, J. Lauwers and P. Taels, On a Wilson lines approach to the study of jet quenching, [arXiv:1307.5518 [hep-ph]].

[12] A. Majumder, Calculating the Jet Quenching Parameter $\hat{q}$ in Lattice Gauge Theory, Phys. Rev. C 87 (2013) 034905 [arXiv:1202.5295 [nucl-th]].

[13] E. Braaten and A. Nieto, Effective field theory approach to high temperature thermodynamics, Phys. Rev. D 51 (1995) 6990 [hep-ph/9501375 ]; Free energy of QCD at high temperature, Phys. Rev. D 53 (1996) 3421 [hep-ph/9510408]. K. Kajantie et al., Generic rules for high temperature 
dimensional reduction and their application to the standard model, Nucl. Phys. B 458 (1996) 90 [hep-ph/9508379].

[14] A. Hietanen et al., Three-dimensional physics and the pressure of hot QCD, Phys. Rev. D 79 (2009) 045018 [arXiv:0811.4664 [hep-lat]].

[15] M. D’Onofrio et al., to appear.

[16] M. Lüscher and P. Weisz, Locality and exponential error reduction in numerical lattice gauge theory, JHEP 0109 (2001) 010 [hep-lat/ 0108014 ] .

[17] K. J. Eskola et al., The Fragility of high-p(T) hadron spectra as a hard probe, Nucl. Phys. A 747 (2005) 511 [hep-ph/0406319] . A. Dainese, C. Loizides and G. Paić, Leading-particle suppression in high energy nucleus-nucleus collisions, Eur. Phys. J. C 38 (2005) 461 [hep-ph/0406201].

[18] X. Ji, Parton Physics on Euclidean Lattice, arXiv:1305.1539 [hep-ph] . H. W. Lin, Calculating the $x$ Dependence of Nucleon Parton Distribution Functions, POS (LATTICE $2013) 293$.

[19] M. Laine and Y. Schröder, Two-loop QCD gauge coupling at high temperatures, JHEP 0503 (2005) 067 [hep-ph/0503061].

[20] A. Mykkänen, The static quark potential from a multilevel algorithm for the improved gauge action, JHEP 1212 (2012) 069 [arXiv:1209.2372 [hep-lat] ] .

[21] G. 't Hooft, A Planar Diagram Theory for Strong Interactions, Nucl. Phys. B 72 (1974) 461. See also B. Lucini and M. Panero, SU(N) gauge theories at large N, Phys. Rept. 526 (2013) 93 [arXiv:1210.4997 [hep-th] ]; Introductory lectures to large-N QCD phenomenology and lattice results, arXiv:1309.3638 [hep-th] ] and M. Panero, Recent results in large-N lattice gauge theories, POS (LATTICE 2012) 010 [arXiv:1210.5510 [hep-lat]] for recent reviews.

[22] B. Lucini, M. Teper and U. Wenger, Properties of the deconfining phase transition in SU(N) gauge theories, JHEP 0502 (2005) 033 [ hep-lat/ 0502003 ]. B. Bringoltz and M. Teper, The Pressure of the SU(N) lattice gauge theory at large-N, Phys. Lett. B 628 (2005) 113 [hep-lat/ 0506034 ] . $\mathrm{S}$. Datta and S. Gupta, Scaling and the continuum limit of the finite temperature deconfinement transition in $S U\left(N_{c}\right)$ pure gauge theory, Phys. Rev. D 80 (2009) 114504 [arXiv: 0909.5591 [hep-lat ] ] . M. Panero, Thermodynamics of the QCD plasma and the large-N limit, Phys. Rev. Lett. 103 (2009) 232001 [arXiv:0907.3719 [hep-lat] ] . A. Mykkänen, M. Panero and $\mathrm{K}$. Rummukainen, Casimir scaling and renormalization of Polyakov loops in large- $N$ gauge theories, JHEP 1205 (2012) 069 [arXiv: 1202.2762 [hep-lat] ] . B. Lucini, A. Rago and E. Rinaldi, $S U\left(N_{c}\right)$ gauge theories at deconfinement, Phys. Lett. B 712 (2012) 279 [arXiv: 1202.6684 [hep-lat] ].

[23] M. Caselle et al., Thermodynamics of SU(N) Yang-Mills theories in 2+1 dimensions I - The confining phase, JHEP 1106 (2011) 142 [arXiv: 1105.0359 [hep-lat] ] ; Thermodynamics of $S U(N)$ Yang-Mills theories in 2+1 dimensions II - The deconfined phase, JHEP 1205 (2012) 135 [arXiv: 1111.0580 [hep-th] ]. P. Bialas et al., Three dimensional finite temperature SU(3) gauge theory near the phase transition, Nucl. Phys. B 871 (2013) 111 [arXiv: 1211.3304 [hep-lat] ]. 